\title{
Validation of Thorpe-scale-derived vertical diffusivities against microstructure measurements in the Kerguelen region
}

\author{
Y.-H. Park ${ }^{1}$, J.-H. Lee ${ }^{2}$, I. Durand ${ }^{1}$, and C.-S. Hong ${ }^{2}$ \\ ${ }^{1}$ MNHN-Sorbonne Universités, UPMC, Univ Paris 06, CNRS-IRD, LOCEAN Laboratory, Muséum National d'Histoire \\ Naturelle, 43, rue Cuvier, 75005 Paris, France \\ ${ }^{2}$ Korea Institut of Ocean Science \& Technology, Ansan, Korea \\ Correspondence to: Y.-H. Park (yhpark@mnhn.fr)
}

Received: 2 June 2014 - Published in Biogeosciences Discuss.: 8 August 2014

Revised: 28 October 2014 - Accepted: 10 November 2014 - Published: 11 December 2014

\begin{abstract}
The Thorpe scale is an energy-containing vertical overturning scale of large eddies associated with sheargenerated turbulence. This study investigates indirect estimates of vertical diffusivities from the Thorpe scale method in the polar front region east of the Kerguelen Islands based on fine-scale density profiles gathered during the 2011 KEOPS2 (KErguelen Ocean and Plateau compared Study 2) cruise. These diffusivities are validated in comparison with diffusivities estimated from the turbulence dissipation rate directly measured via a TurboMAP (Turbulence ocean Microstructure Acquisition Profiler) microstructure profiler. The results are sensitive to the choice of the diffusivity parameterization and the overturn ratio $R_{\mathrm{O}}$, and the optimal results have been obtained from the parameterization by Shih et al. (2005) and the $R_{\mathrm{O}}=0.25$ criterion, rather than the parameterization by Osborn (1980) and the $R_{\mathrm{O}}=0.2$ criterion originally suggested by Gargett and Garner (2008).

The Thorpe-scale-derived diffusivities in the KEOPS2 region show a high degree of spatial variability, ranging from a canonical value of $O\left(10^{-5}\right) \mathrm{m}^{2} \mathrm{~s}^{-1}$ in the Winter Water layer and in the area immediately north of the polar front to a high value of $O\left(10^{-4}\right) \mathrm{m}^{2} \mathrm{~s}^{-1}$ in the seasonal thermocline between the surface mixed layer and the Winter Water. The latter high diffusivities are found especially over the shallow plateau southeast of the Kerguelen Islands and along the polar front that is attached to the escarpment northeast of the islands. The interaction of strong frontal flow with prominent bottom topography likely causes the observed elevated mixing rates.
\end{abstract}

\section{Introduction}

Vertical mixing is a dominant factor in controlling vertical fluxes of heat, salt, and nutrients, so the estimation of vertical (or diapycnal) diffusivities especially in the upper layer of the ocean was one of the primary priorities of the physical component of the KEOPS2 (KErguelen Ocean and Plateau compared Study 2) cruise. During the cruise, direct turbulence measurements were made at selected stations using a tethered, quasi-freefall profiler, TurboMAP, measuring the microstructure of velocity shear. An indirect method for estimating vertical diffusivities using more accessible CTD (conductivity-temperature-depth) density profiles is the Thorpe scale method (Thorpe, 1977). The objective of this study is to estimate the vertical diffusivities from finescale density profiles using the Thorpe scale method and validate them in comparison with microstructure measurements collected via a TurboMAP during the KEOPS2 cruise.

The performance of the Thorpe scale method compared to microstructure estimates has been known to depend on the stratification of the water column and surface environment conditions affecting the ship motion. While good agreement between the two methods has been reported in lowlatitude regions of high stratification and low winds (Ferron et al., 1998; Klymak et al., 2008), the application of the Thorpe scale method in the Southern Ocean could be compromised because of low stratification and extreme environments (Frants et al., 2013). The latter authors reported that the CTD-based fine-structure methods overestimate microstructure diffusivities by 1 to 2 orders of magnitude in the 
southeastern Pacific and Drake Passage, claiming their real limitations in the Southern Ocean.

Another intriguing issue concerns the existence of two different parameterizations of vertical diffusivity $K$ in terms of turbulence dissipation rate $\varepsilon$ and buoyancy frequency $N$. Note that $N^{2}=-\left(g / \rho_{0}\right) \rho_{z}$, where $g$ is gravity, $\rho_{0}$ is a constant reference density, and $\rho_{z}$ is a vertical gradient of potential density calculated at each depth over a vertical extent of $10 \mathrm{~m}$.

For example, Osborn (1980) suggested a well-known parameterization as

$K=0.2 \varepsilon / N^{2}$.

On the other hand, Shih et al. (2005) proposed a new parameterization for the energetic turbulence regime $\left(\varepsilon / \nu N^{2}>100\right)$ based on the laboratory and numerical experiments as

$K=2 v\left(\varepsilon / v N^{2}\right)^{1 / 2}$,

where $v=(1.5$ to 1.8$) \times 10^{-6} \mathrm{~m}^{2} \mathrm{~s}^{-1}$ is the kinematic viscosity in the temperature range of 0 to $5^{\circ} \mathrm{C}$ and $\varepsilon / \nu N^{2}$ is the turbulence intensity parameter. Note that, for the moderate turbulence intensity regime $\left(7<\varepsilon / v N^{2}<100\right)$, the parameterization of $K$ by Shih et al. (2005) is same as Eq. (1) proposed by Osborn (1980).

While the TurboMAP measurements lead to direct estimates of $\varepsilon$, the Thorpe scale method gives its indirect estimates by making use of an empirical relationship between the Thorpe scale and $\varepsilon$. These two (direct and indirect) estimates of $\varepsilon$ can be applied to the above two parameterizations of $K$, yielding a total of four kinds of $K$ estimates (Osborn_ $\varepsilon$, Osborn_Thorpe, Shih_E, Shih_Thorpe) at each station of intercomparison. Because of their utmost importance, the detailed procedures for the preliminary processing of CTD data as well as for the detection and validation of overturns for calculating the Thorpe scale are given in Sect. 2. These are largely based on a comprehensive paper by Gargett and Garner (2008), although we have added some modifications. We will show in Sect. 3 that the results are sensitive to the choice of the $K$ parameterization and to the criteria of the overturn validation. In Sect. 4 we present vertical diffusivities in the KEOPS2 area estimated from the optimally chosen parameterization and overturn ratio. Discussion of a displacement shape method recently proposed by van Haren and Gostiaux (2014) is given in Sect. 5, followed by conclusions.

\section{Preliminary processing of CTD data}

CTD data used for deriving fine-scale density profiles were collected during the October-November 2011 KEOPS2 cruise aboard the R/V Marion Dufresne in the polar front (PF) region east of the Kerguelen Islands (see Park et al., 2014 , for details of the regional hydrography and frontal circulation). Here we used a total of 22 CTD profiles gathered using two sets of Sea-Bird Electronics (SBE) 9plus sensors at stations shown in Fig. 1, where the concomitant TurboMAP stations are shown circled. The CTD profiles mostly extend from the surface to the bottom, while the TurboMAP measurements mostly extend from the surface to about $400 \mathrm{~m}$, limiting our validation of the Thorpe scale method to the upper $400 \mathrm{~m}$.

A critical step to a successful validation of the latter method resides in the minimization of the effects of instrument noise and measurement errors, which may be due to the conductivity cell's thermal lag, pressure reversals due to ship roll, and salinity spiking caused by the differing time responses of the temperature and conductivity sensors (e.g. Gargett and Garner, 2008). A series of procedures for processing CTD data are given below.

1. For minimizing thermal lag arising from the conductivity cell thermal mass effects, the raw CTD data have been first processed using the Sea-Bird processing software (http://www.seabird.com/sites/default/ files/documents/SBEDataProcessing_7.23.2.pdf). The data-processing module "Cell Thermal Mass" performs conductivity thermal mass correction, for which we used typical values $(\alpha=0.03 ; 1 / \beta=7.0)$ recommended for SBE 9plus in the above software.

2. Salinity spiking, which can be caused by misalignment of temperature and conductivity with each other, was removed on acquisition from a pre-programmed SBE 9 plus deck unit by advancing conductivity by $0.073 \mathrm{~s}$. Therefore, there was no need to run the data-processing module "Align CTD".

3. Due to the effect of the ship heave motion on the hardcoupled CTD, the fall speed of CTD continuously varies while scanning and can occasionally reverse sign for short periods. We located segments of pressure reversals and edited out the data between successive encounters of the same pressure, although this can be also done via the data-processing module "Loop Edit".

4. At this stage, the CTD conductivity and salinity data were corrected with water bottle salinity previously analyzed using a salinometer.

5. In order to further minimize any spike-like anomalies in property (salinity, potential temperature, potential density) profiles, we applied a quadratic fit to successive $10 \mathrm{~m}$ segments to detect and discard "extremely abnormal" anomalies surpassing 4 times the root mean square (rms) anomaly relative to the fitting curve. About $0.03 \%$ of total scans are eliminated by this process.

6. Our final CTD data processing consisted of averaging and subsampling profiles at regular depth intervals. For this, we averaged the property profiles over a $10 \mathrm{~cm}$ window that is centered at each depth incremented by a regular span of $10 \mathrm{~cm}$. On average, about two to three scans 
(a)

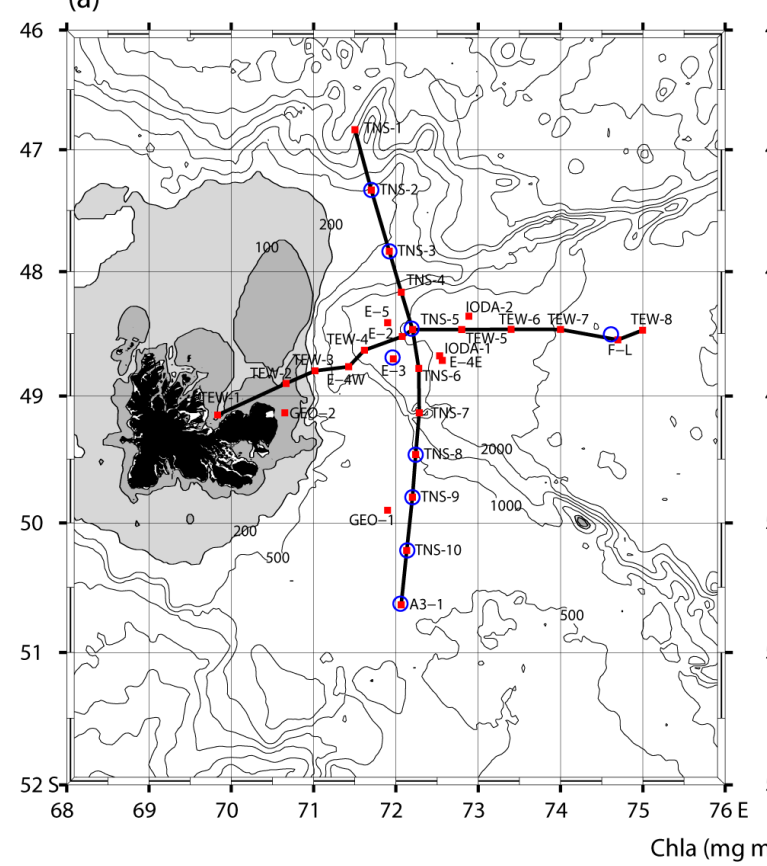

(b)

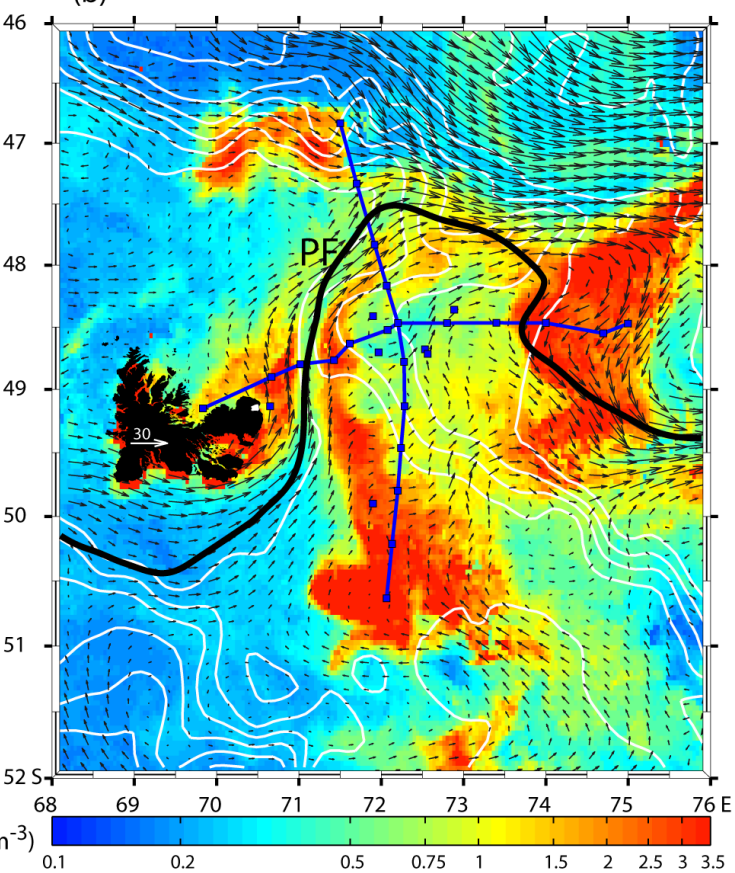

Figure 1. (a) Map showing the KEOPS2 CTD stations (red dots) on or close to two N-S and E-W transects superimposed on the detailed bathymetry. The concomitant TurboMAP microstructure profiler stations are indicated by blue circles. Isobaths greater than $500 \mathrm{~m}$ are given every $500 \mathrm{~m}$ and the seabed shallower than $200 \mathrm{~m}(100 \mathrm{~m}$ ) is lightly (darkly) shaded. (b) These stations are also superimposed on a representative satellite image of chlorophyll concentration (colors) and a surface geostrophic velocity field (arrows) constructed from the combined data sets from altimetry and trajectories of drifters launched during the cruise. The geographical position of the polar front (PF) is indicated. This figure (b) has been adapted from Park et al. (2014).

enter into this $10 \mathrm{~cm}$ averaging, which is roughly consistent with a mean fall rate of $\sim 0.9 \mathrm{~m} \mathrm{~s}^{-1}$ of our $24 \mathrm{~Hz}$ CTD. This filters out any high-frequency random noise of a length scale less than $10 \mathrm{~cm}$; thus the smallest detectable overturn should be of $20 \mathrm{~cm}$ in vertical extent. Note also that most density profiles start from $20 \mathrm{~m}$ below the sea surface because the near-surface measurements are often found to be very contaminated, probably by turbulence generated by the hull. These processed density profiles form our basic data set used in the following section.

\section{Thorpe scale analysis}

\subsection{Thorpe scale and vertical diffusivity}

A first step for detecting overturns generated by turbulence in a stratified water column consists of sorting a potential density profile $\rho(z)$, which may contain inversions, into a stable monotonic sequence without inversions. The vertical displacement necessary for generating the stable profile is the Thorpe displacement $d$, and the Thorpe scale $L_{\mathrm{T}}$ is defined as the rms of $d$ within each overturn that is a depth range over which the sum of $d$ drops back to 0 (Dillon, 1982).
A classical measure of the overturning length is the Ozmidov scale $L_{\mathrm{O}}$ (Ozmidov, 1965) defined as

$\varepsilon=L_{\mathrm{O}}^{2} N^{3}$.

Dillon (1982) suggested a linear relationship between $L_{\mathrm{T}}$ and $L_{\mathrm{O}}$, such that

$L_{\mathrm{O}}=0.8( \pm 0.4) L_{\mathrm{T}}$,

although an exact linear relation between these two overturning scales cannot be expected due to spatial and temporal variability of the turbulent field (Ferron et al., 1998).

Inserting Eqs. (3) and (4) into Eqs. (1) and (2), the vertical diffusivity can be estimated indirectly from $L_{\mathrm{T}}$ as

$K=0.128 L_{\mathrm{T}}^{2} N$,

according to the Osborn parameterization, and as

$K=1.6 v^{1 / 2} L_{\mathrm{T}} N^{1 / 2}$,

according to the Shih parameterization. We give below a series of procedures necessary for an optimal estimation of $L_{\mathrm{T}}$, and thus of $K$ from the Thorpe scale method. 


\subsection{Determination of a threshold noise level of density}

The major concern in the overturn validation is whether the identified overturns are false overturns associated with random noise and/or residual effects of salinity spiking. To prevent false overturns due to random noise, we followed an intermediate density profile method proposed by Gargett and Garner (2008), who modified a profile processing method of Ferron et al. (1998). The Gargett and Garner method tracks only significant differences in the density profile, where a significant difference is defined relative to a threshold noise level below which a density difference is considered as due to random noise. For this purpose, we have calculated the rms of detrended density anomalies over successive $10 \mathrm{~m}$ segments for selected "well-mixed" layers within the cruise data set. This yielded a mean value of $1.75 \times 10^{-4} \mathrm{~kg} \mathrm{~m}^{-3}$. We considered a multiple of 4 of the latter value, $7 \times 10^{-4} \mathrm{~kg} \mathrm{~m}^{-3}$, as our threshold noise level. Note that the latter value is close to $5 \times 10^{-4} \mathrm{~kg} \mathrm{~m}^{-3}$ of Gargett and Garner (2008), who applied instead a multiple of 5 to a slightly smaller mean rms density anomaly of $1.0 \times 10^{-4} \mathrm{~kg} \mathrm{~m}^{-3}$ obtained in the Ross Sea region using a SBE 9plus CTD.

\subsection{Creation of an intermediate density profile}

Once the threshold value is determined as above, building an intermediate density profile is straightforward as clearly explained in Gargett and Garner (2008). In short, an intermediate profile is created first from the top to the bottom, maintaining a constant density until a density change greater than the threshold value. A similar profile starting from the bottom to the top is also created, and a final intermediate profile used here is the average of the two individual (downward and upward) profiles. An example of this procedure for determining an intermediate density profile is shown in Fig. 2.

\subsection{Validation of overturns}

Gargett and Garner (2008) reviewed several previous methods of overturn validation and proposed a practical method using an overturn ratio, $R_{\mathrm{O}}=\min \left(L^{+} / L, L^{-} / L\right)$, where $L$ is the total vertical extent of an overturn and $L^{+}\left(L^{-}\right)$is the cumulative extent occupied by positive (negative) Thorpe displacements. These authors found the $T-S$ tightness method suggested by Galbraith and Kelly (1996) unsatisfactory and did not recommend any further rejection based on any measure of $T-S$ tightness. Gargett and Garner (2008) reasoned that a single perfect overturn sampled straight through the middle would contain equal lengths (extents) of positive and negative displacements (or $R_{\mathrm{O}}=0.5$ ), and suggested a critical $R_{\mathrm{O}}$ value of 0.2 , below which the prospect overturn is suspected of being caused by residual salinity spiking.

We found that the $R_{\mathrm{O}}=0.2$ criterion is not sufficient in our case, but the use of $R_{\mathrm{O}}=0.25$ at least is rather necessary to detect the false overturns associated with suspicious den-

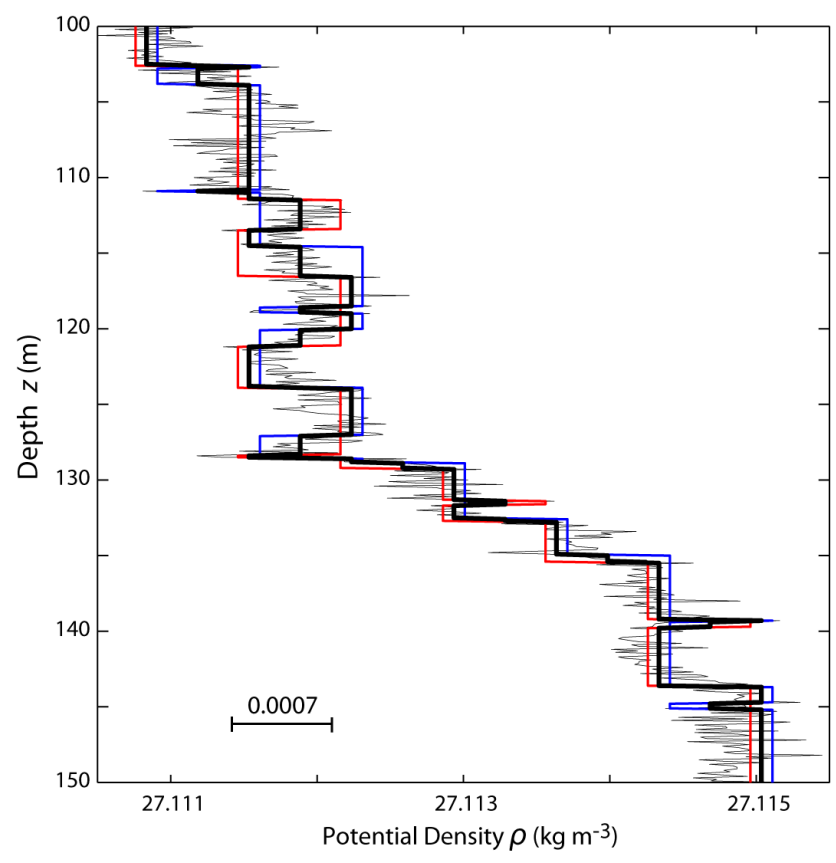

Figure 2. Sample section of intermediate profiles generated from the top (red), from the bottom (blue), and from the average of these two (thick black) of a measured density profile (thin black), following the method of Gargett and Garner (2008). The threshold density noise $\left(0.0007 \mathrm{~kg} \mathrm{~m}^{-3}\right)$ used is indicated.

sity spiking. An example is given in Fig. 3 for station A3-1, where we observe four clear density spikes as indicated by red arrows. The overturns associated with first two spikes near 200 and $225 \mathrm{~m}$ have $R_{\mathrm{O}}$ values between 0.2 and 0.25 (Fig. 3c), and thus can be considered as false overturns according to the $\left(R_{\mathrm{O}}=\right) 0.25$ criterion, whereas the 0.2 criterion might have validated them as true overturns. The third spike just above $300 \mathrm{~m}$ has a $R_{\mathrm{O}}$ value much smaller than 0.2 and thus can be easily discriminated as a false overturn even by the more stringent 0.2 criterion. The fourth spike just below $300 \mathrm{~m}$ reveals a $R_{\mathrm{O}}$ value so close to 0.25 that the 0.25 criterion appears to be absolutely necessary for invalidating the prospect overturn. In summary, all four suspected overturns can be safely discriminated as false overturns by our new criterion $R_{\mathrm{O}}=0.25$, whereas the previously proposed $R_{\mathrm{O}}=0.2$ criterion by Gargett and Garner (2008) fails to detect these false overturns, except for the one associated with the third spike just above $300 \mathrm{~m}$. We will show below that these four suspicious overturns really correspond to false overturns.

\subsection{Sensitivity of the vertical diffusivity to its parameterization}

With the Thorpe scales $L_{\mathrm{T}}$ obtained based on the $R_{\mathrm{O}}=$ 0.25 criterion, we have calculated vertical diffusivities from the Osborn parameterization (Eq. 5) and the Shih parameterization (Eq. 6), denoted hereafter as $K_{\mathrm{O}_{-} \mathrm{T}}$ and $K_{\mathrm{S}_{-} \mathrm{T}}$, 
(a) Potential Density

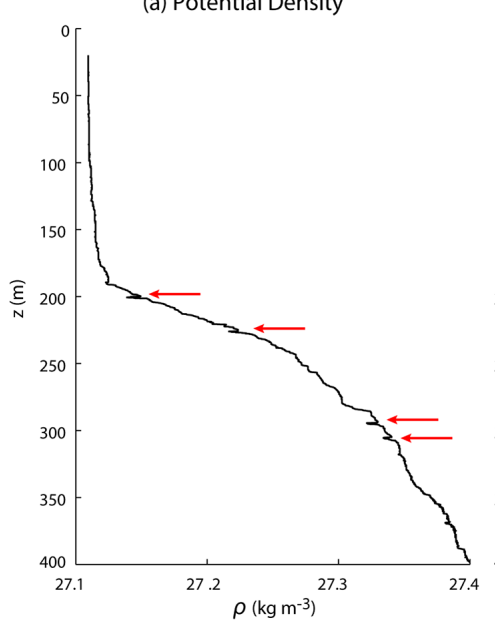

(b) Thorpe Scale

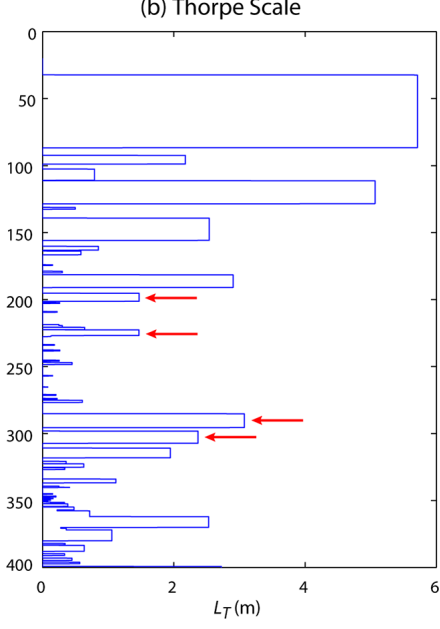

(c) Overturn Ratio

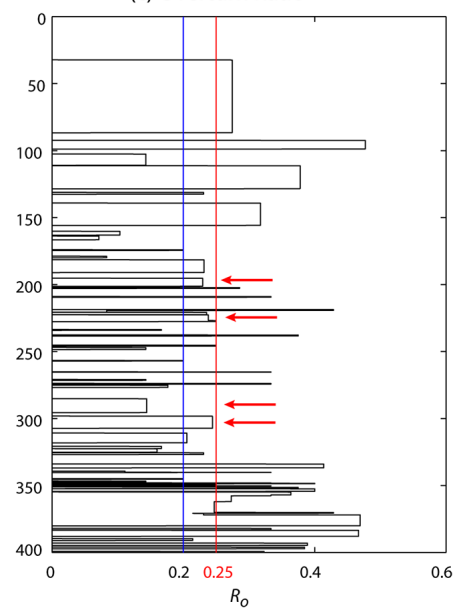

Figure 3. Sample illustration showing the (a) intermediate density profile, (b) Thorpe scales, and (c) overturn ratios calculated at station A3-1. Four suspicious false overturns associated with abnormal spikes clearly apparent in the density profile are indicated by red arrows. Two criteria of overturn validation are shown by colored vertical lines in (c) blue for $R_{\mathrm{O}}=0.2$ and red for $R_{\mathrm{O}}=0.25$.

(a) Potential Density

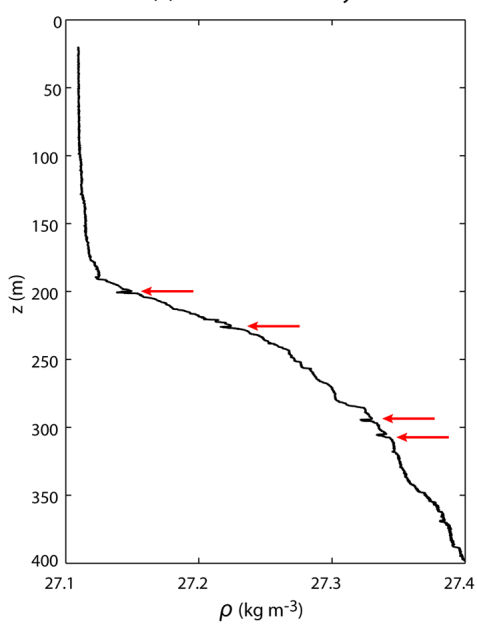

(b) $R_{0}=0.2$

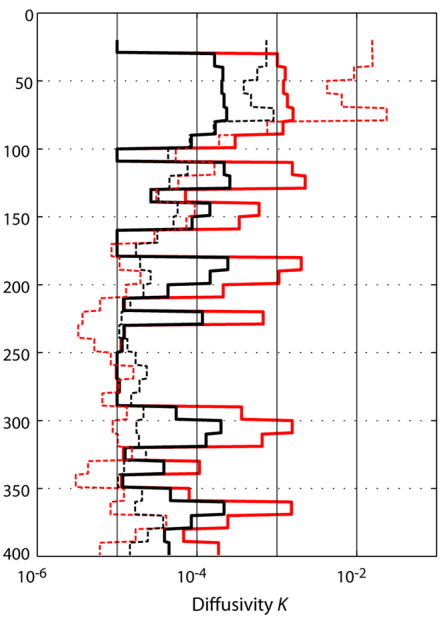

(c) $R_{0}=0.25$

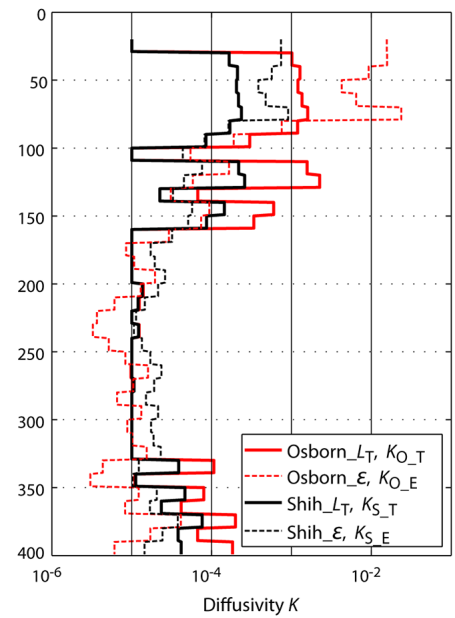

Figure 4. Different diffusivity profiles at station A3-1 calculated with different pairs of parameterization (Osborn or Shih) and observational method (Thorpe scale $L_{\mathrm{T}}$ or TurboMAP-derived $\varepsilon$ ) using the two overturn validation criteria of (b) $R_{\mathrm{O}}=0.2$ and (c) $R_{\mathrm{O}}=0.25$. Note that the four abnormal spikes seen in (a), being the repetition of Fig. 3a, give rise to great overestimation in the Thorpe-scale-derived diffusivities with $R_{\mathrm{O}}=0.2$, but this feature disappears completely with $R_{\mathrm{O}}=0.25$.

respectively. The regions where no overturns are detected do not necessarily mean no vertical mixing, as already remarked by Ferron et al. (1998), but our method cannot resolve tiny overturns smaller than $20 \mathrm{~cm}$, as mentioned in Sect. 2. In this case the corresponding diffusivities are set to $1 \times 10^{-5} \mathrm{~m}^{2} \mathrm{~s}^{-1}$, a value corresponding to the minimum value $\left(1.024 \times 10^{-5} \mathrm{~m}^{2} \mathrm{~s}^{-1}\right)$ of the TurboMAP-derived diffusivities estimated using the Shih parameterization. The resulting diffusivities have been averaged over regular vertical intervals of $10 \mathrm{~m}$. These Thorpe-scale-derived diffusivities at station A3-1 are compared in Fig. 4 with those calculated according to Eqs. (1) and (2) using the TurboMAP- derived $\varepsilon$, denoted hereafter as $K_{\mathrm{O}-\mathrm{E}}$ and $K_{\mathrm{S} \_\mathrm{E}}$, respectively (Fig. 4 c). We also show in the same figure $K_{\mathrm{O}_{-} \mathrm{T}}$ and $K_{\mathrm{S} \_\mathrm{T}}$ estimated using the $R_{\mathrm{O}}=0.2$ criterion, always in comparison with $K_{\mathrm{O} \_\mathrm{E}}$ and $K_{\mathrm{S} \_\mathrm{E}}($ Fig. $4 \mathrm{~b}$ ). Several interesting features appear. First, the Thorpe-scale-derived diffusivities for the case of $R_{\mathrm{O}}=0.2$ are systematically overestimated compared to the TurboMAP-derived diffusivities by up to 1 to 2 orders of magnitude in the layer below $80 \mathrm{~m}$, while the converse is true above it in the surface mixed layer. Second, such an overestimation or underestimation in the Thorpescale-derived diffusivities is much more pronounced by an order of magnitude with the Osborn parameterization $\left(K_{\mathrm{O}_{-} \mathrm{T}}\right)$ 

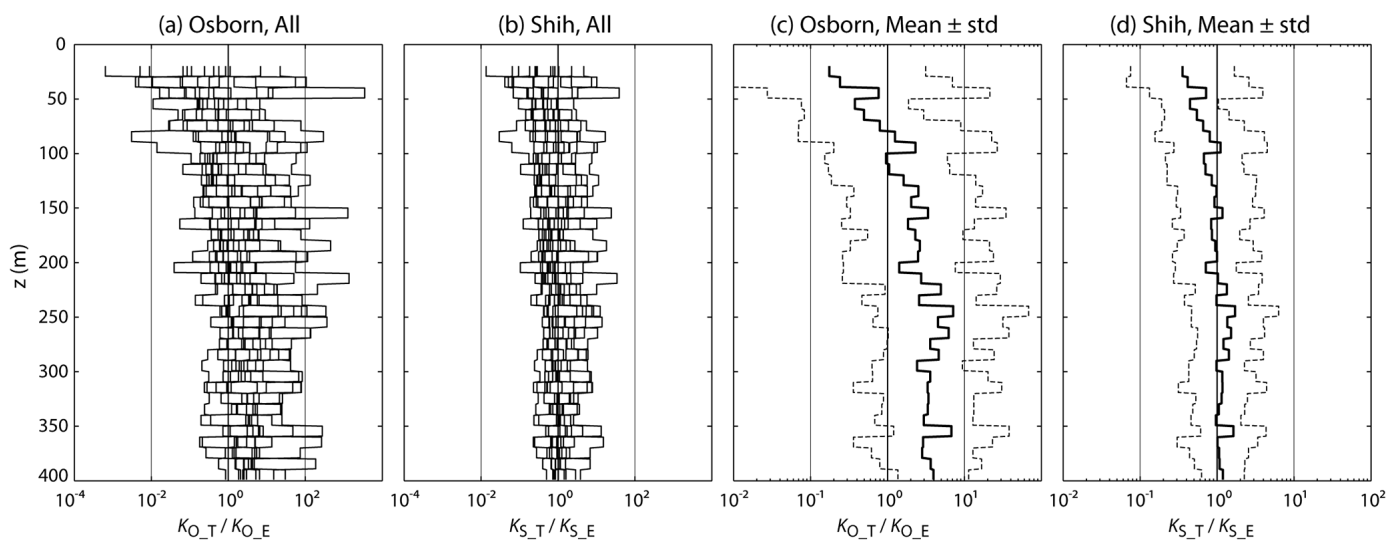

Figure 5. Ratio profiles of the Thorpe-scale-derived diffusivities and the TurboMAP-derived diffusivities at all intercomparison stations based on (a) the Osborn parameterization and (b) the Shih parameterization. Here, the $R_{\mathrm{O}}=0.25$ criterion is commonly used. (c) and (d) are the same as (a) and (b) but for the mean (black) and standard deviation (grey) of all stations.

compared to the Shih parameterization $\left(K_{\mathrm{S}_{-} \mathrm{T}}\right)$. Third, the TurboMAP-derived diffusivities from both parameterizations $\left(K_{\mathrm{O} \_\mathrm{E}}\right.$ and $\left.K_{\mathrm{S} \_\mathrm{E}}\right)$ agree with each other within a factor of 2 , on average, except for the surface mixed layer where the difference reaches up to an order of magnitude. As compared to $K_{\mathrm{S} \_\mathrm{E}}$, there appears to be a tendency of great overestimation (slight underestimation) of $K_{\mathrm{O} \_\mathrm{E}}$ in the surface mixed layer (deeper layer below $80 \mathrm{~m}$ ). Finally, we observe the efficiency of our new overturn validation criterion, $R_{\mathrm{O}}=0.25$ (see Fig. 4c), which yields a much closer agreement with different estimates at the abovementioned four suspicious false overturns, while the $R_{\mathrm{O}}=0.2$ criterion (see Fig. $4 \mathrm{~b}$ ) still yields there abnormal overestimation in the Thorpe-scalederived diffusivities (as compared to microstructure diffusivities). This confirms our previous conviction that the four suspected overturns represent really false overturns which escape from detection with $R_{\mathrm{O}}=0.2$ but can be safely detected with $R_{\mathrm{O}}=0.25$. We have verified similar features in several other stations too, and we will use hereafter uniquely the $R_{\mathrm{O}}=0.25$ criterion for the detection of false overturns.

In order to statistically evaluate the sensitivity of the vertical diffusivity to its parameterization, we have calculated for all intercomparison stations and depths the ratio of the Thorpe-scale-derived diffusivities and the TurboMAPderived diffusivities, separately using the Osborn parameterization $\left(K_{\mathrm{O}_{-} \mathrm{T}} / K_{\mathrm{O}_{-} \mathrm{E}}\right)$ and the Shih parameterization $\left(K_{\mathrm{S}_{-} \mathrm{T}} / K_{\mathrm{S} \_\mathrm{E}}\right)($ Fig. 5$)$. There is a clear tendency of overestimation by the Osborn parameterization, especially in the layer deeper than $100 \mathrm{~m}$, by up to 2 orders of magnitude or more (Fig. 5a). This is much less evident with the Shih parameterization which shows a comparatively very compact variability of ratio within an order of magnitude around unity (Fig. 5b). On the other hand, in the surface layer above $100 \mathrm{~m}$ there is an increasing negative tendency toward the surface for both parameterizations, as already mentioned. This is probably due to a very low stratification of the surface mixed layer, which prevents detection of moderate overturns whose density differences are smaller than our threshold noise level of $7 \times 10^{-4} \mathrm{~kg} \mathrm{~m}^{-3}$.

Assuming a log-normal distribution of diffusivity ratios $R_{\mathrm{dif}}$, the mean and standard deviation (SD) of $\log \left(R_{\mathrm{dif}}\right)$ have been used to represent the basic statistics of $R_{\text {dif }}$. With the Osborn parameterization (Fig. 5c), the Thorpe-scalederived diffusivities below $200 \mathrm{~m}$ overestimate (compared to the TurboMAP-derived diffusivities) by a mean $R_{\text {dif }}$ of $\sim 4$, with a $( \pm 1 \mathrm{SD})$ variability range of $(0.7,20)$, on average. The overestimation gradually diminishes toward the surface and changes its sign near $80 \mathrm{~m}$ to show a near-surface peak of underestimation, with a mean $R_{\text {dif }}$ of $\sim 0.2(0.01,5)$. In contrast to this, the Shih parameterization (Fig. $5 \mathrm{~d}$ ) yields a much more reasonable agreement, with a mean $R_{\text {dif }}$ close to unity $(0.3,3)$ over most of the water column, except for the surface layer, which always shows a general but somewhat reduced tendency of underestimation by $\sim 0.4(0.1,2)$. Consequently, we conclude that the use of the Shih parameterization, rather than the Osborn parameterization, is highly desirable in the estimation of vertical diffusivities for our study area, which is also worthy of testing its broad applicability in the other sectors of the Southern Ocean.

\section{Thorpe-scale-derived vertical diffusivities in the KEOPS2 area}

For all KEOPS2 density profiles, we have estimated the Thorpe-scale-derived vertical diffusivities $K$ by applying the overturn ratio criterion $R_{\mathrm{O}}=0.25$ and the Shih parameterization. Figure $6 \mathrm{a}$ and $\mathrm{b}$ represent the spatial distribution of $K$ in the upper $400 \mathrm{~m}$ along the approximately north-south $(\mathrm{N}-\mathrm{S})$ - and east-west (E-W)-oriented transects (see Fig. 1 for the position of stations). The $50 \mathrm{~m}$ depth-averaged $K$ values are given in Table 1. Care is warranted when citing the values for the top $50 \mathrm{~m}$ depth range because of the abovemen- 
Table 1. Fifty-meter averaged vertical diffusivities (in $10^{-5} \mathrm{~m}^{2} \mathrm{~s}^{-1}$ ) at KEOPS2 stations estimated from the Thorpe scale method using the Shih parameterization and the $R_{\mathrm{O}}=0.25$ criterion.

\begin{tabular}{lrrrrrrrr}
\hline Station & \multicolumn{7}{c}{ depth range $(\mathrm{m})$} \\
\cline { 2 - 9 } & $0-50$ & $50-100$ & $100-150$ & $150-200$ & $200-250$ & $250-300$ & $300-350$ & $350-400$ \\
\hline TNS-10 & 44 & 37 & 34 & 2 & 6 & 1 & 5 & 2 \\
TNS-9 & 22 & 5 & 1 & 4 & 2 & 1 & 6 & 19 \\
TNS-8 & 18 & 8 & 1 & 8 & 1 & 3 & 6 & 7 \\
TNS-7 & 2 & 2 & 1 & 1 & 2 & 2 & 12 & 5 \\
TNS-6 & 34 & 11 & 7 & 4 & 2 & 6 & 3 & 2 \\
TNS-5 & 16 & 18 & 13 & 1 & 9 & 9 & 5 & 2 \\
TNS-4 & 67 & 19 & 5 & 1 & 1 & 11 & 1 & 2 \\
TNS-3 & 16 & 22 & 27 & 2 & 2 & 3 & 6 & 1 \\
TNS-2 & 1 & 1 & 1 & 1 & 1 & 5 & 1 & 1 \\
TNS-1 & 1 & 4 & 3 & 1 & 1 & 3 & 1 & 1 \\
TEW-1 & 20 & 4 & NaN & NaN & NaN & NaN & NaN & NaN \\
TEW-2 & 1 & 1 & NaN & NaN & NaN & NaN & NaN & NaN \\
TEW-3 & 1 & 4 & 2 & 3 & 1 & 21 & 2 & 1 \\
TEW-4 & 1 & 2 & 5 & 4 & 2 & 2 & 7 & 2 \\
TEW-5 & 1 & 6 & 1 & 3 & 2 & 8 & 4 & 4 \\
TEW-6 & 1 & 2 & 7 & 1 & 2 & 4 & 1 & 5 \\
TEW-7 & 1 & 1 & 7 & 1 & 1 & 2 & 3 & 2 \\
TEW-8 & 2 & 4 & 1 & 2 & 2 & 11 & 7 & 9 \\
A3-1 & 13 & 18 & 13 & 2 & 1 & 1 & 2 & 5 \\
E-2 & 4 & 1 & 3 & 6 & 1 & 2 & 6 & 2 \\
E-4W & 9 & 19 & 24 & 14 & 19 & 7 & 1 & 9 \\
F-L & 6 & 5 & 6 & 18 & 2 & 3 & 6 & 3 \\
\hline
\end{tabular}

tioned underestimation tendency; multiplication by 2 to 3 is recommended instead. The $K$ distribution is highly heterogeneous in both the vertical and horizontal directions, varying from a low level of $<2 \times 10^{-5} \mathrm{~m}^{2} \mathrm{~s}^{-1}$ (areas with no color shading) mostly in the Winter Water and the layer below to a relatively high level of $>10^{-4} \mathrm{~m}^{2} \mathrm{~s}^{-1}$ (areas encircled by white lines) observed predominantly in the upper $150 \mathrm{~m}$. The area-averaged mixing rate in the subsurface layer (200$400 \mathrm{~m}$ ) over the entire KEOPS2 area is $4 \times 10^{-5} \mathrm{~m}^{2} \mathrm{~s}^{-1}$, a value close to recent estimates from microstructure measurements in a similar layer $(250-500 \mathrm{~m})$ north of the Kerguelen Plateau by Waterman et al. (2013). It is also of the same order of magnitude as strain-derived diffusivities from Argo float profiles in the same area (Whalen et al., 2012; Wu et al., 2011). For comparison, somewhat contrasting results have been reported in the PF region of Drake Passage; elevated subsurface diffusivities of $O\left(10^{-4}\right) \mathrm{m}^{2} \mathrm{~s}^{-1}$ have been estimated from Thorpe scales and CTD strain by Thompson et al. (2007), whereas direct microstructure measurements of turbulence levels by St. Laurent et al. (2012) have rather revealed a much weaker background level of $O\left(10^{-5}\right) \mathrm{m}^{2} \mathrm{~s}^{-1}$ (see also Waterhouse et al., 2014), similar to the estimates in the Kerguelen region.

The spatial $K$ distribution appears to have some correlation with the regional frontal circulation carrying different water masses. For example, in the N-S transect (Fig. 6a) the areas of elevated diffusivities are mostly confined in the seasonal thermocline $(50-150 \mathrm{~m})$ above the Winter Water $\left(T_{\min }<2{ }^{\circ} \mathrm{C}\right)$ developed to the south of the PF, with the exception being over the continental slope east of the Kerguelen Islands (TNS-7 to TNS-9) where the mixing rate is low. The strongest diffusivities are found over the shallow plateau $(\sim 600 \mathrm{~m})$ southeast of the islands (TNS-10 and A3-1) and close to the PF over the northern escarpment northeast of the islands (TNS-3 to TNS-5). Our results are consistent with similar previous results showing enhanced turbulent levels in the regions where deep-reaching strong flow meets a rugged or abrupt bottom topography (Wu et al., 2011; St. Laurent et al., 2012; Whalen et al., 2012; Waterman et al., 2013; Waterhouse et al., 2014). On the other hand, the Winter Water layer $(150-250 \mathrm{~m})$ generally coincides with the layer of diffusivity minimum. Also, the mixing rate in warmer waters north of the PF (TNS-1, TNS-2) is quite low throughout the upper $400 \mathrm{~m}$, resting close to its background level of $O\left(10^{-5}\right) \mathrm{m}^{2} \mathrm{~s}^{-1}$.

The diffusivity estimates at station A3-1 are similar in vertical structure to, but smaller in magnitude by a factor of 4 than, those estimated at the same station during the 2005 KEOPS1 cruise (Park et al., 2008). Several factors may explain this difference. First, in Park et al. (2008) the discrimination of false from true overturns was based on the criterion that a minimum density difference of $0.0015 \mathrm{~kg} \mathrm{~m}^{-3}$ (or 
(a) N-S Transect

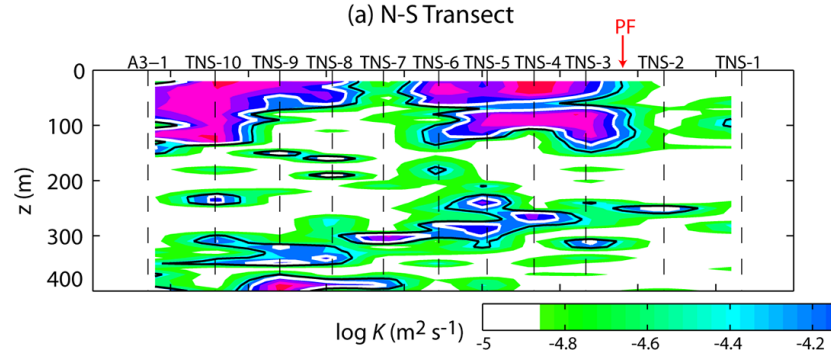

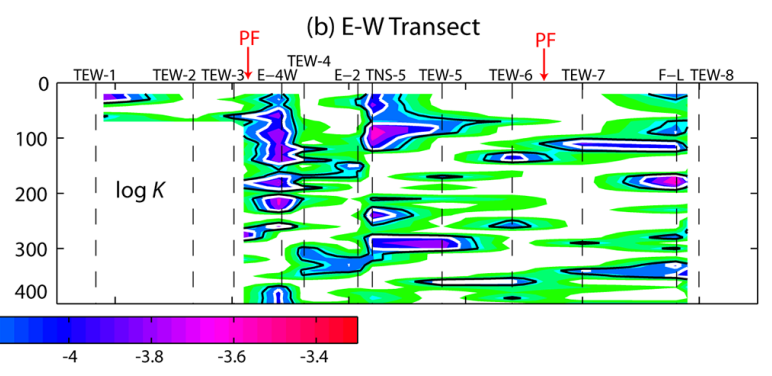$$
\text { . }
$$

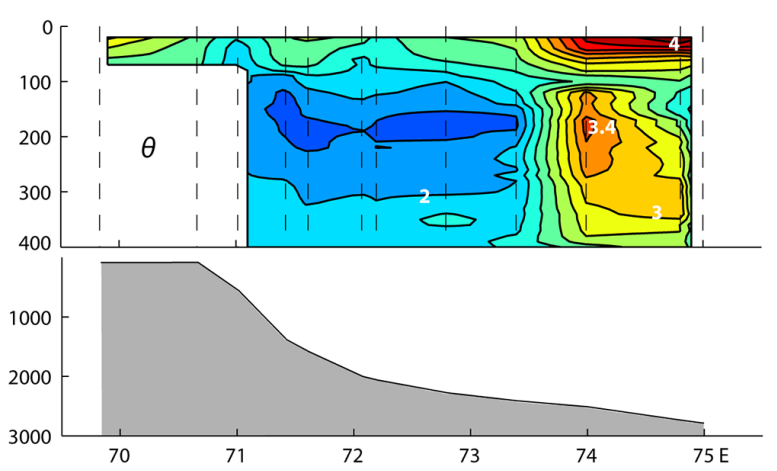

Figure 6. Thorpe-scale-derived diffusivity sections (calculated using the Shih parameterization and the $R_{\mathrm{O}}=0.25$ criterion) of the upper $400 \mathrm{~m}$ on (a) the $\mathrm{N}-\mathrm{S}$ transect and (b) the E-W transect (top panels; see Fig. 1 for locations of the transects and stations). Diffusivity $K$ values, which range from $1 \times 10^{-5}$ to $7 \times 10^{-4} \mathrm{~m}^{2} \mathrm{~s}^{-1}$, are shown in $\log (K)$. White and black lines correspond to $1 \times K=10^{-4} \mathrm{~m}^{2} \mathrm{~s}^{-1}$ and $K=5 \times 10^{-5} \mathrm{~m}^{2} \mathrm{~s}^{-1}$, respectively, while the regions without color shading correspond to $K<2 \times 10^{-5} \mathrm{~m}^{2} \mathrm{~s}^{-1}$. For ease of interpretation in combination with the 3-D frontal circulation of water masses (see also Fig. 1), corresponding temperature sections (middle panels) and seabed profiles drawn from in situ station depths measured during the KEOPS2 cruise (bottom panels) are also shown.

3 times the estimated noise level of $0.0005 \mathrm{~kg} \mathrm{~m}^{-3}$ ) is necessary to validate an overturn. As will be seen later in the Discussion section, such a density difference criterion is inefficient at discriminating the false overturns associated with density spikes. Therefore, the latter criterion tends to overestimate the mixing rates as compared to the overturn ratio criterion, which is found to be agreeably efficient, especially with $R_{\mathrm{O}}=0.25$ (see Figs. 3, 4). Second, Park et al. (2008) used the Osborn parameterization, which is found to yield mean diffusivities significantly higher by a factor of 4 compared to the Shih parameterization adapted in the present study (see Fig. 5).

On the E-W transect (Fig. 6b), the spatial distribution of $K$ is quite complex compared to the $\mathrm{N}-\mathrm{S}$ section, and there does not appear any simple pattern that can be easily connected to the frontal circulation of water masses. Nevertheless, we remark a relatively strong mixing rate of $O\left(10^{-4}\right) \mathrm{m}^{2} \mathrm{~s}^{-1}$ over much of the water column at station E-4W that is located close to the northward-flowing PF along the escarpment east of the Kerguelen Islands, while the weakest rate of $O\left(10^{-5}\right) \mathrm{m}^{2} \mathrm{~s}^{-1}$ is observed at station TEW-7, where warmer polar frontal zone waters flow southward (Park et al., 2014) along with the southward-retroflecting PF (see also Fig. 1b). Other stations in the section show a highly undulating vertical structure with a moderate mixing rate less than $5 \times 10^{-5} \mathrm{~m}^{2} \mathrm{~s}^{-1}$, in general.

\section{Discussion and conclusions}

\subsection{Comparison with a displacement shape method}

Recently, van Haren and Gostiaux (2014) suggested a new method of discriminating various overturns and intrusions via inspection of displacement $(d)$ shapes in a $d-z$ plane. They showed that, depending on the displacement slopes $z / d$, the true overturns can be categorized into different types of vortex, such as, most frequently, half-turn Rankine vortices $(1 / 2<z / d<1)$ and, rather rarely, full-turn Rankine vortices $(z / d \sim 1)$ or solid-body rotations $(z / d=1 / 2)$. These authors recommended to use temperature profiles rather than density profiles if salinity-compensating intrusions are negligible, because the density profiles are much noisier thus cause an overestimate of turbulence parameters. They mentioned also that more-or-less equivalent results (within a factor of 1.5) may be obtained with the density data only by imposing a limit of discarding density variations smaller than $1 \times 10^{-3} \mathrm{~kg} \mathrm{~m}^{-3}$, twice the expected noise level.

In our case of the upper layer of the Antarctic zone the temperature is not an adequate parameter for investigating overturns because of its unstable vertical distribution, with a gradual temperature increase with depth from the Winter Water $\left(T_{\min }<2^{\circ} \mathrm{C}\right)$ centered at about $200 \mathrm{~m}$ to the Upper Circumpolar Deep Water $\left(T_{\max } \sim 2.3{ }^{\circ} \mathrm{C}\right)$ centered at about $700 \mathrm{~m}$ (Park et al., 2014). Then, we have tested the method 

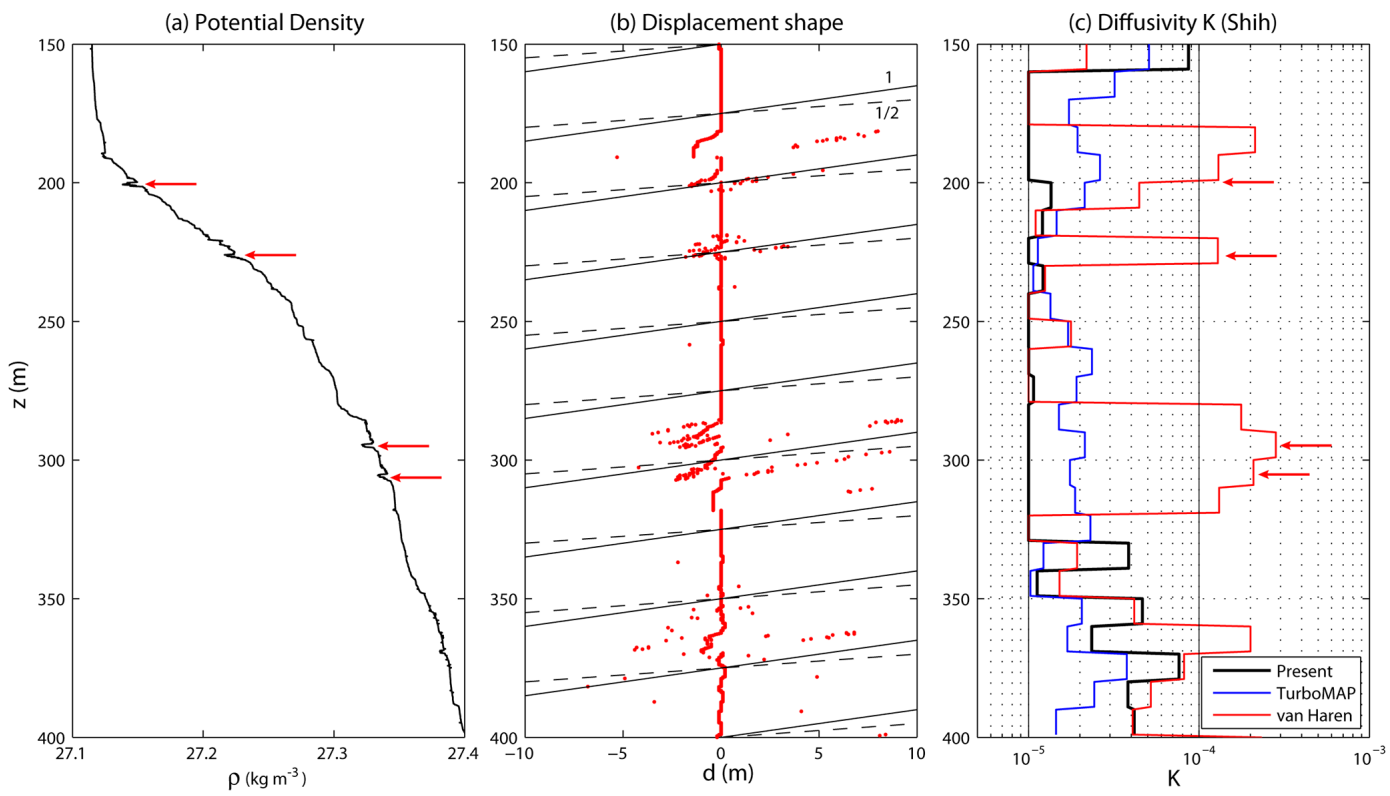

Figure 7. (a) Potential density profile in the 150-400 m layer at station A3-1, with four clear density spikes being indicated by red arrows. (b) Displacement points (red dots) computed from corrected density data after discarding density variations smaller than $1 \times 10^{-3} \mathrm{~kg} \mathrm{~m}^{-3}$. Displacement slopes $z / d=1$ (solid) and $z / d=1 / 2$ (dashed) are superimposed. (c) Diffusivity profile estimated from the displacement shape method (red) in comparison with those from our best approach (black) and TurboMAP data (blue), all using the Shih parameterization. See the text for more details. Red arrows indicate the location of the density spikes seen in (a).

using corrected density profiles after discarding density variations (relative to sorted density profiles) smaller than the proposed limit of $1 \times 10^{-3} \mathrm{~kg} \mathrm{~m}^{-3}$ by van Haren and Gostiaux (2014).

An example of the test is given in Fig. 7 for station A31, already discussed in Figs. 3 and 4, where there exist four clear density spikes (red arrows). van Haren and Gostiaux (2014) previously remarked that discarding density variations $<1 \times 10^{-3} \mathrm{~kg} \mathrm{~m}^{-3}$ unfortunately limits the use of investigating the shape of displacements. Consistent with this remark, discriminating various types of overturns by inspection of displacement shapes does not appear very obvious (Fig. 7b). Nevertheless, we observe that the most significant displacements appear mostly in the vicinity of the above four density spikes, with a rather marked asymmetry between positive and negative displacements. As before, the mixing rates have been estimated using the Shih parameterization (Eq. 6) and the Thorpe scales $L_{\mathrm{T}}$ of identified overturns. The red line in Fig. 7c illustrates the resultant diffusivities averaged over intervals of $10 \mathrm{~m}$, in comparison with those from our best approach of the Thorpe scale method (using intermediate density profiles and applying the overturn ratio criterion $R_{\mathrm{O}}=0.25$ and the Shih parameterization: black line) and the TurboMAP measurements (blue line). Note that the latter two lines are borrowed from Fig. 4c. Compared to our best approach and the TurboMAP data, the displacement shape method yields in many places comparable diffusivities within a factor of 2 , but with a great exception in the vicinity of the above four density spikes, where we observe a significant overestimation (relative to the TurboMAP data) by as much as an order of magnitude. This indicates that, in great contrast to our approach, the displacement shape method is not able to discriminate the false overturns associated with apparent density spikes (caused probably by a mismatch between the temperature and conductivity sensors), the major cause of most false overturns in the oceans (e.g., Galbraith and Kelley, 1996; Gargett and Garner, 2008).

\subsection{Concluding remarks}

We have validated the Thorpe-scale-derived vertical diffusivities in the PF region east of the Kerguelen Islands using more direct estimates from the TurboMAP microprofiler measurements at selected stations during the KEOPS2 cruise. We have emphasized the need for a careful treatment of raw CTD data to obtain density profiles as fine as possible but with a maximum removal of random noise and measurement errors. Here we have used density profiles on $10 \mathrm{~cm}$ scales, which can yield fine-resolution diffusivities at scales up to $10 \mathrm{~m}$ after a suitable vertical averaging. This vertical resolution is far finer by an order of magnitude compared to other indirect strain and shear methods that use a vertical integration scale of the order of $200 \mathrm{~m}$ (e.g., Thompson et al., 2007; Frants et al., 2013). A compelling argument for obtaining such finely resolved diffusivities from the Thorpe scale method may be that they should provide, as compared to coarser estimates from the strain and shear methods, de- 
tailed local information useful for precisely evaluating the vertical fluxes of nutrients and other biogeochemical materials across the seasonal thermocline.

Our comparative results are found to be sensitive to the choice of the parameterization of diffusivity and the overturn validation criteria. The use of the Shih parameterization (Eqs. 2 and 6) combined with our overturn ratio criterion of $R_{\mathrm{O}}=0.25$ has yielded significantly better results by a factor of 5 compared to the results from the Osborn parameterization (Eqs. 1 and 5) and the $R_{\mathrm{O}}=0.2$ criterion suggested by Gargett and Garner (2008). The latter criterion $\left(R_{\mathrm{O}}=0.2\right)$ appears to be insufficient (too low) to detect most false overturns associated with apparent density spikes, thus overestimating diffusivities. Moreover, the Osborn parameterization is shown to be much more sensitive to such an overestimation compared to the Shih parameterization. This study demonstrates that the Thorpe scale method remains a useful tool for investigating the fine-scale diffusivities in the Southern Ocean if one makes judicious use of the combined Shih parameterization and $R_{\mathrm{O}}=0.25$ criterion. This is in stark contrast to Frants et al. (2013), who claimed the real limitations of the CTD-based fine-structure methods to be in Drake Passage and the eastern Pacific sector of the Southern Ocean.

The Thorpe-scale-derived vertical diffusivities in the KEOPS2 region vary from a background level of $O\left(10^{-5}\right) \mathrm{m}^{2} \mathrm{~s}^{-1}$ in the Winter Water layer to a relatively high level of $O\left(10^{-4}\right) \mathrm{m}^{2} \mathrm{~s}^{-1}$ in the seasonal thermocline, which is a transitional boundary layer between the Winter Water and the surface mixed layer. The latter high-diffusivity feature is especially pronounced at stations over the shallow plateau southeast of the Kerguelen Islands and on the cold side of the PF running along the escarpment northeast of the islands. This is consistent with the general belief that the interaction of strong flow with rough or abrupt bottom topography produces high internal wave energy and intensified turbulence (e.g., Ferron et al., 1998; Klymak et al., 2008; St. Laurent et al., 2012; Waterman et al., 2013). On the other hand, at stations immediately north of the PF where warmer surface waters are encountered, diffusivity values are particularly low.

Acknowledgements. This work was supported by the French research program of INSU-CNRS LEFE-CYBER (Les enveloppes fluides et l'environnement - Cycles biogéochimiques, environnement et ressources), the French ANR (Agence Nationale de la Recherche, SIMI-6 program, ANR-10-BLAN-0614), the French CNES (Centre National d'Etudes Spatiales), and the French Polar Institute IPEV (Institut Polaire Paul-Emile Victor). We thank the captain and crew of the R/V Marion Dufresne for their professional contributions to various field experiments during the 2011 KEOPS2 cruise. We are also grateful to S. Blain, project manager, and B. Queguiner, chief scientist, for their great effort in making the KEOPS2 cruise successful. We thank two anonymous reviewers for constructive comments which have significantly improved the original manuscript. All co-authors of the present paper have benefited by the STAR program, a French-Korean research collaboration program, for their mutual exchanges in 2012 and 2013. J.-H. Lee was partially supported by the National Research Foundation of Korea Grant funded by the Korean government (NRF-2009-C1AAA001-0093065) to participate in the KEOPS2 cruise.

Edited by: S. Blain

\section{References}

Dillon, T. M.: Vertical overturns: A comparison of Thorpe and Ozmidov length scales, J. Geophys. Res., 87, 9601-9613, 1982.

Ferron, B., Mercier, H., Speer, K., Gargett, A., and Polzin, K.: Mixing in the Romanche fracture zone, J. Phys. Oceanogr., 28, 19291945, 1998.

Frants, M., Damerell, G. M., Gille, S. T., Heywood, K. J., MacKinnon, J., and Springtall, J.: An assessment of densitybased fine-scale methods for estimating diapycnal diffusivity in the Southern Ocean, J. Atmos. Ocean. Tech., 30, 2647-2661, doi:10.1175/jtech-d-12-00241.1, 2013.

Galbraith, P. S. and Kelley, D. E.: Identifying overturns in CTD profiles, J. Atmos. Ocean. Tech., 13, 688-702, 1996.

Gargett, A. and Garner, T.: Determining Thorpe scales from shiplowered CTD density profiles, J. Atmos. Ocean. Tech., 25, 16571670, doi:10.1175/2008JTECHO541.1, 2008.

Klymak, J. M., Pinkel, R., and Rainville, L.: Direct breaking of the internal tide near topography: Kaena Ridge, Hawaii, J. Phys. Oceanogr., 38, 380-399, doi:10.1175/2007JPO3728.1, 2008.

Osborn, T. R.: Estimates of the local rate of vertical diffusion from dissipation measurements, J. Phys. Oceanogr., 10, 83-89, 1980.

Ozmidov, R. V.: On the turbulent exchange in a stably stratified ocean, Izv. Acad. Sci., USSR Atmos. Oceanic Phys., 1, 853-860, 1965.

Park, Y.-H., Fuda, J. L., Durand, I., and Naveira Garabato, A. C.: Internal tides and vertical mixing over the Kerguelen Plateau, Deep-Sea Res. Pt. II, 55, 582-593, 2008.

Park, Y.-H., Durand, I., Kestenare, E., Rougier, G., Zhou, M., d'Ovidio, F., Cotté, C., and Lee, J.-H.: Polar Front around the Kerguelen Islands: An up-to-date determination and associated circulation of surface/subsurface waters, J. Geophys. Res.Oceans, 119, 6575-6592, doi:10.1002/2014JC010061, 2014.

Shih, L. H., Koseff, J. R., Ivey, G. N., and Ferziger, J. H.: Parameterization of turbulent fluxes and scales using homogeneous sheared stably stratified turbulence simulations, J. Fluid Mech., 525, 193214, 2005.

St. Laurent, L. C., Naveira Garabato, A. C., Ledwell, J. R., Thurnherr, A. M., Toole, J. M., and Watson, A. J.: Turbulence and diapycnal mixing in Drake Passage, J. Phys. Oceanogr., 42, 21432152, doi:10.1175/JPO-D-12-027.1, 2012.

Thompson, A. F., Gille, S. T., MacKinnon, J. A., and Sprintall, J.: Spatial and temporal patterns of small-scale mixing in Drake Passage, J. Phys. Oceanogr., 37, 572-592, doi:10.1175/JPO3021.1, 2007.

Thorpe, S. A.: Turbulence and mixing in a Scottish Loch, Philos. Trans. Roy. Soc. London, 286A, 125-181, 1977.

van Haren, H. and Gostiaux, L.: Characterizing turbulent overturns in CTD-data, Dyn. Atmos. Ocean, 66, 58-76, 2014. 
Waterhouse A. F., MacKinnon, J. A., Nash, J. D., Alford, M. H., Kunze, E., Simmons, H. L., Polzin, K. L., St. Laurent, L. C., Sun, O. M., Pinkel, R., Talley, L. D., Whalen, C. B., Hussen, T. N., Carter, G. S., Fer, I., Waterman, S., Naveira Garabato, A. C., Sanford, T. B., and Lee, C. M.: Global pattern of diapycnal mixing from measurements of the turbulent dissipation rate, J. Phys. Oceanogr., 44, 1854-1872, doi:10.1175/JPO-D-13-0104.1, 2014.

Waterman, S., Naveira Garabato, A. C., and Polzin, K. L.: Internal waves and turbulence in the Antarctic Circumpolar Current, J. Phys. Oceanogr., 43, 259-282, doi:10.1175/JPO-D-11-0194.1, 2013.
Whalen, C., Talley, L. D., and MacKinnon, J. A.: Spatial and temporal variability of global ocean mixing inferred from Argo profiles, Geophys. Res. Lett., 39, L18612, doi:10.1029/2012GL053196, 2012.

Wu, L. X., Jing, Z., Riser, S., and Visbeck, M.: Seasonal and spatial variations of Southern Ocean diapycnal mixing from Argo profiling floats, Nat. Geosci., 4, 363-366, doi:10.1038/ngeo1156, 2011. 